\title{
DIAGNOSIS OF ORF VIRUS INFECTION IN SHEEP AND GOATS BY VIRUS ISOLATION, POLYMERASE CHAIN REACTION AND SEQUENCING
}

\author{
Srinivasa Babu $\mathrm{T}^{1}$, Rathnamma $\mathrm{D}^{1 *}$, Shrikrishna Isloor ${ }^{1}$, Chandranaik $\mathrm{BM}^{2}$, Veeregowda $\mathrm{BM}^{1}$, \\ Manjunath Reddy GB $^{3}$
}

\footnotetext{
${ }^{1}$ Department of Veterinary Microbiology, Veterinary College, KVAFSU, Hebbal, Bangalore 560 024, India

${ }^{2}$ Institute of Animal Health and Veterinary Biologicals, Hebbal, Bengaluru-560 024

${ }^{3}$ National Institute of Veterinary Epidemiology and Disease Informatics, Yelahanka, Benagaluru-64
}

Received - August 29, 2017; Revision - October 27, 2017; Accepted - January 31, 2018

Available Online - February 20, 2018

DOI: http://dx.doi.org/10.18006/2018.6(1).176.187

KEYWORDS
ORF virus
Virus isolation
PCR
Phylogeny
Sheep and goats

\begin{abstract}
Outbreaks of ORF virus (ORFV) infection in small ruminants, which occurred in eight districts of Karnataka, India were investigated during this study. Seventy scab samples were collected from affected sheep and goats from 27 outbreaks and subjected for virus isolation and polymerase chain reaction. The ORFV was successfully isolated in the chicken embryos (CE) by CAM route of inoculation. Polymerase chain reaction (PCR) was standardized targeting B2L gene for detection of ORFV. The screening of 70 samples for B2L gene amplification revealed 60 per cent positivity of Orf infection. The phylogenetic analysis based on nucleotide and deduced amino acid sequences of full length and partial B2L gene revealed that, the ORFV isolates had highest genetic homology among them and with the other Indian ORFV isolates. The maximum genetic heterogeneity was observed with ORFV isolates from other countries. Further, full length B2L gene sequence based analysis provided significant information about the genetic relationship among the various ORFV isolates. Sequence analysis revealed high nucleotide (98.7 -100 per cent) and amino acids (98.4-99.7 per cent) identity with Indian isolates maximum with Assam/09 \& Assam/10 ORFV isolates.
\end{abstract}

* Corresponding author

E-mail: rathnarohit@gmail.com (Rathnamma D)

Peer review under responsibility of Journal of Experimental Biology and Agricultural Sciences.

Production and Hosting by Horizon Publisher India [HPI] (http://www.horizonpublisherindia.in/).

All rights reserved.
All the article published by Journal of Experimental Biology and Agricultural Sciences is licensed under a Creative Commons Attribution-NonCommercial 4.0 International License Based on a work at www.jebas.org.

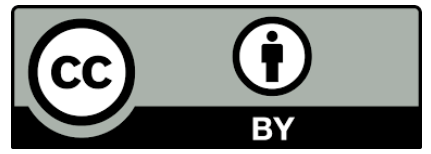




\section{Introduction}

'ORF' which is Old English meaning 'rough' also known as contagious ecthyma, sore and scabby mouth disease, contagious pustular dermatitis, infectious pustular dermatitis, affecting mainly sheep and goats causing significant economic losses in the livestock production. The disease also reported to infect wild ruminants and other mammals. The disease is distributed world wide and endemic in many countries wherever sheep and goats are raised. The disease also has a zoonotic potential affecting the people mainly farmers, veterinarians and butchers who are in direct contact with infected animals especially during shearing, docking, drenching and slaughtering or indirect contact with infected animals (Bora et al., 2011; Spyrou \& Valiakos, 2015; Gelaye et al., 2016; Caravaglio \& Khachemoune, 2017). The disease is highly proliferative and often self limiting. Clinically, Orf disease progresses from erythema to macule, papule, vesicle formation and then pustules to thick crusts called scabs. The scabs are often friable and mild trauma causes the lesions to bleed easily. These lesions are commonly found on muzzle, lips, around the mouth, oral mucosa, ears and around the nostrils. The lesions can also be seen on feet, eyelids and teats. Young animals are severely affected, preventing them from suckling. Severely affected animals may lose their weight and becoming more susceptible for secondary bacterial infections. Morbidity is very high in young animals and mortality is usually low. Sheep are susceptible to reinfection and chronic infection also occur (Gelaye et al., 2016). The disease is transmitted by direct contact or through exposure to the contaminated feeding troughs and fomites. The Orf disease is caused by Orf virus (ORFV), a Parapoxvirus (PPV) genus belongs to the family Poxviridae. Orf virus is robust in dry environment and it is clear that orf outbreaks occur through direct contact with infectious materials in the environment and after this it shows systemic spread (McKeever et al., 1987). The Orf virus has a linear double stranded DNA of approximately $135 \mathrm{~kb}$ in size. The complete genome sequences of ORFV and other parapoxviruses have been published (Delhon et al., 2004). The genes that are required for viral replication are conserved in the central region of the viral genome and sequence variation is found in the terminal ends of the viral genome which encode the molecules required for virushost cell interactions. The B2L gene of virus is a major envelope gene which encodes an envelope protein of $45 \mathrm{kDa}$, a highly immunogenic protein. The B2L gene has been used for detection of ORFV by PCR and molecular epidemiological analysis of ORFV infections (Inoshima et al., 2000; Hosamani et al., 2007). An emerging feature of PPV is that it individually exhibits a highly expanding host range and can also infect humans. The present study was aimed at characterizations of Orf virus isolates based on the B2L gene sequences analysis.

\section{Materials and Methods}

\subsection{Collection of samples}

A total of 70 scab samples were collected from sheep and goats from the eight districts of Karnataka which have dense sheep population (Fig.1, 2 and 3). The samples were collected in viral transport medium (VTM) and were processed as per the procedure described by OIE (2007).

\subsection{Isolation of Orf virus in embryonated chicken eggs (ECE)}

The 11 day old embryonated chiken eggs were procured from University Poultry farm, Veterinary College, Hebbal, Bengaluru. Processed scab samples were inoculated onto chorio-allantoic membrane (CAM) of 11 day old chick embryos following the standard protocol as per OIE (2007). Inoculated embryos and control embryos were incubated at $37^{\circ} \mathrm{C}$ in the presence of humidity with periodical shacking and observed daily by candling. After 5 days, the allantoic fluids and CAM were collected carefully. Chorioallantoic membranes were examined for the presence of the characteristic pock lesions.

\subsection{Standardization of Polymerase chain reaction}

The DNA was isolated from the processed samples using QIAamp DNA Mini Kit (catalog no. 51306, Qiagen Pvt. Ltd) and final elution of DNA was done in $30 \mu 1$ of elution buffer and stored at $20^{\circ} \mathrm{C}$ until use. Primers for PCR were synthesized. Primer 1 OVB2LF1 (5'-TCCCTGAAGCCCTATTATTTTTGTG -3') corresponded to positions 560-583 of the B2L gene, while primer 2, OVB2LR1 (5'- GCTTGCGGGCGTTCGGACCTT C-3') corresponded to antisense strand at positions 1138-1115 with an amplicon size of $1137 \mathrm{bp}$ were used in this study, these are as per the Hosamani et al.(2006).

Polymerase chain reaction amplification was carried out in a final reaction volume of $25 \mu \mathrm{l}$ under the PCR conditions of initial denaturation step of $94{ }^{\circ} \mathrm{C}$ for 3 min followed by 29 cycles of 94 ${ }^{\circ} \mathrm{C}$ for $1 \mathrm{~min}, 52{ }^{\circ} \mathrm{C}$ for $1 \mathrm{~min}, 72{ }^{\circ} \mathrm{C}$ for $1 \mathrm{~min}$ and final extension of $72{ }^{\circ} \mathrm{C}$ for $7 \mathrm{~min}$. The PCR products were analyzed by $1.0 \%$ agarose gel electrophoresis. The amplicons were gel purified using MinElute gel extraction kit (QIAGEN) as per manufacturer's protocol.

\subsection{Nucleotide sequencing and phylogenetic analysis}

The purified PCR products were sequenced at M/s Amnion Biotech Pvt Ltd, Bengaluru. Sequences were edited and consensus sequences assembled in Bio Edit (http://www.mbio.ncsu.eduibioedit/bioedit.html) software application. The datasets that are representative of all worldwide variants and datasets from NCBI GenBank (B2Lgene) were 
utilized. The sequences were analysed and determined by Clustal W method in the MegAlign program of Lasergene ((DNASTAR Inc. Madison, USA) (Thompson et al., 1994). The sequences were aligned and phylogenetic tree for B2L gene of ORFV were constructed using MEGA 6.06 software (Kumar et al., 2004). The genetic distances between sequences were calculated and used for construction of a neighbor joining tree for B2L gene (Saitou \& Nei,1987)

\section{Results}

\subsection{Collection of samples}

The areas of suspected field outbreaks of ORF were selected based on endemicity and geographical distribution of the disease (Figure 3). Most of the animals in unorganized farms were showing varied degrees of clinical forms of ORFV infection. Among these, few animals had lesions near eyes and ear regions and few had severe inflammation around the oral cavity. The clinical symptoms included blisters early in the infection and then crusty scabs. Sores were typically found on the lips, muzzle, mouth and also on the eye and the ears with mild pyrexia (Figure 1 and 2). The oral lesions rendered most ailing animals unable to feed, and deaths were noticed in few cases in lambs and kid. However, some animals recovered upon proper treatment.

\subsection{Isolation of Orf virus in embryonated chicken eggs}

In the present study, Orf virus isolation was carried out from 11 day old ECE as per methods described in OIE (2007). The 100 $\mu \mathrm{l}$ of processed scab samples was inoculated by chorio-allantoic membrane $(\mathrm{CAM})$ route. These inoculated eggs were incubated with regulated parameters for 5 days. After fifth day of incubation, allantoic fluids and CAM were harvested as per standard protocol (Figure $4 \mathrm{a} \& \mathrm{~b}$ ). Negative control was inoculated with sterile PBS (Figure 4c). Out of eight samples, six produced characteristic white, firm and necrotic pock lesions on the CAM (Figure 4d). Confirmation of these pock lesions was done by subjecting to PCR with B2L gene amplification of DNA extracted from the pock lesions on the CAM using commercial Qiagen tissue extraction kit. Later on, this amplified product was subjected to agarose gel electrophoresis and clear amplicon of $1137 \mathrm{bp}$ was visualized (Figure 5).

\subsection{Screening of scab samples for B2L gene of ORFV by Polymerase chain reaction}

A number of experiments were performed to optimize the conventional PCR protocol, including concentration of reagents, the template DNA and thermal cycling conditions. The optimized PCR assay was established using a total volume of 25 $\mu 1$. After standardization of different components of PCR, DNA
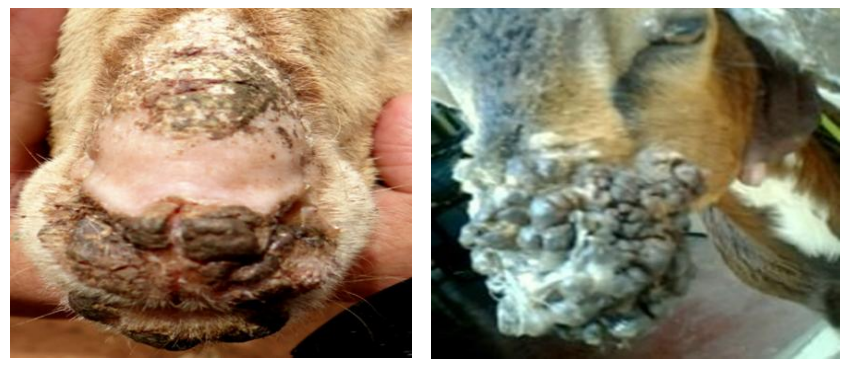

Figure1 Scab lesions around the mouth in sheep and goats
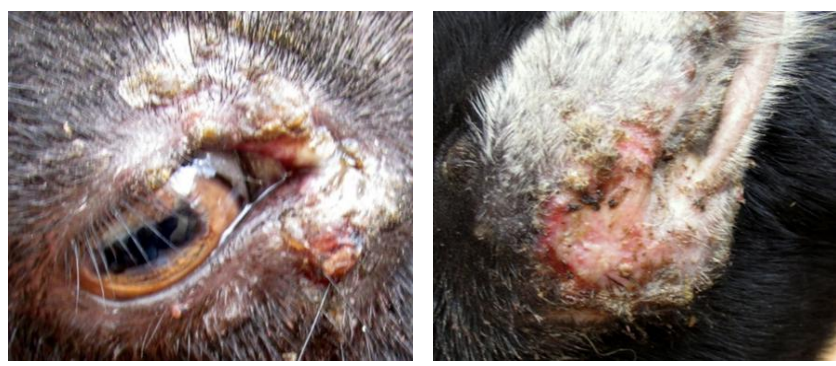

Figure 2 Lesions around eye and on the ear in goats

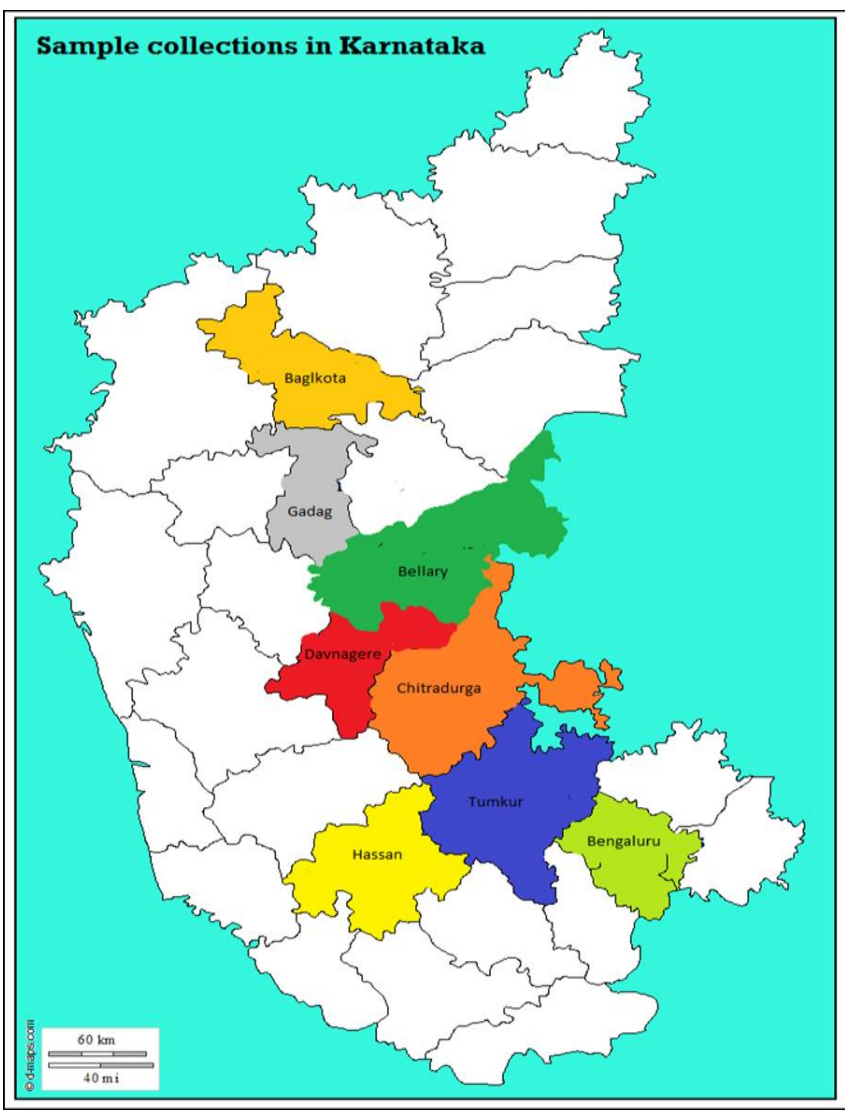

Figure 3 District wise sample collections 


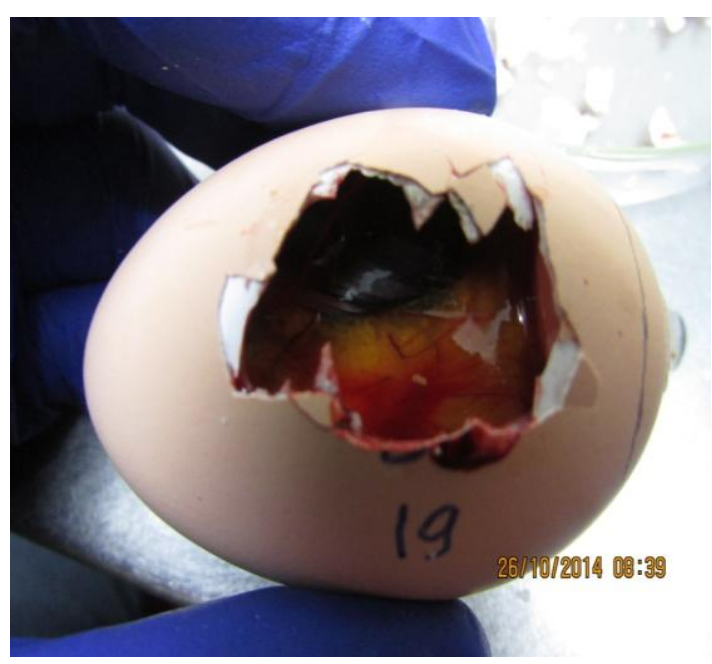

4a. 11 days old embryonated chiken egg

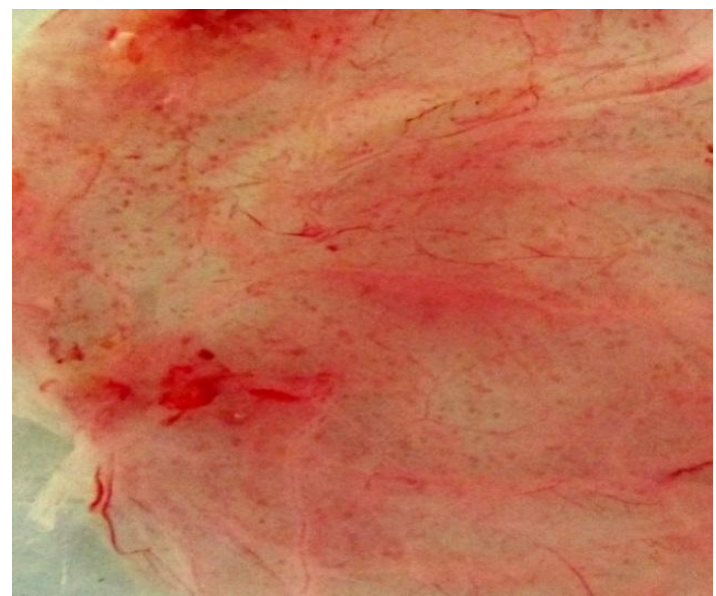

4c Healthy CAM

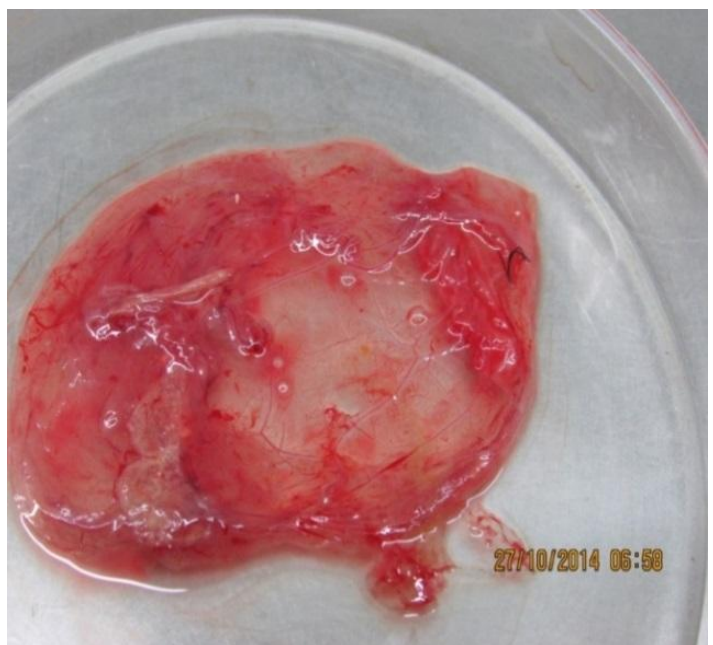

4b. CAM collected from egg

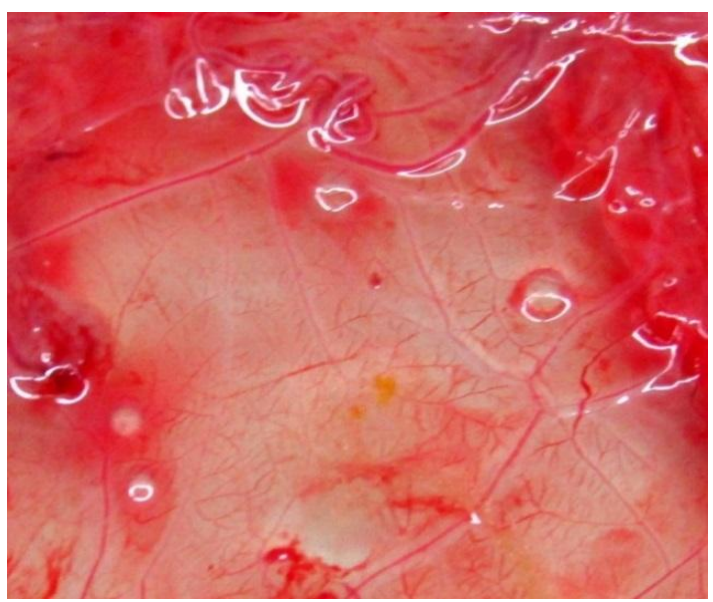

4d Pock lesions on CAM

Figure 4 Pock lesions of ORF virus in embryonated chicken eggs

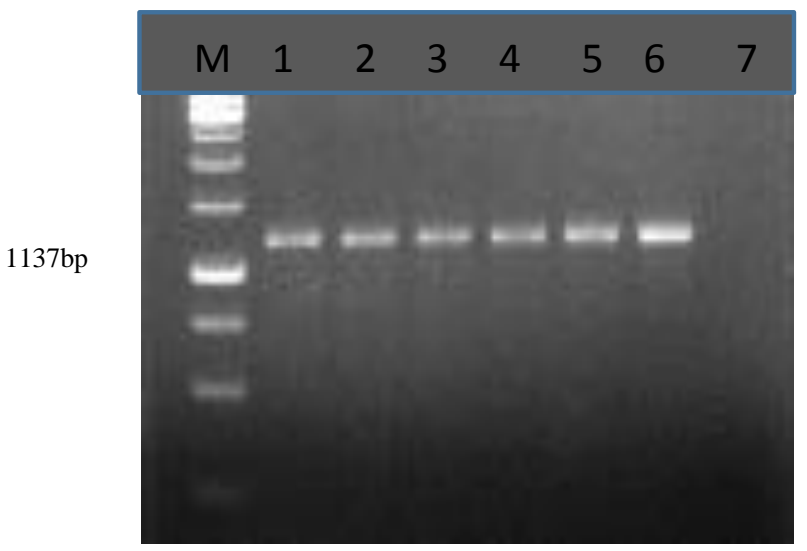

Lane M: 250bp DNA ladder

Lane 1: ORFV KVAFSU VMC -05

Lane 2: ORFV KVAFSU VMC -20

Lane 3: ORFV KVAFSU VMC -51

Lane 4: ORFV KVAFSU VMC -59

Lane 5: ORFV KVAFSU VMC -63

Lane 6: Positive control (ORFV)

Lane 7: Negative control (Sheep pox virus)

Figure 5 PCR amplification of B2L gene of Orf virus

Journal of Experimental Biology and Agriculture Science http://www.jebas.org 
extracted from 70 scab samples were subjected to PCR for detection of B2L gene of ORFV. The amplified products were subjected to agarose gel electrophoresis and clear amplicon of $1137 \mathrm{bp}$ was visualized (Figure 5).

In the present study, out of 70 samples from 27 outbreaks, 42 samples $(60 \%)$ were positive for amplification of B2L gene of ORFV. In Tumkur district, 12 out of 21 samples were positive (57.1\%) by PCR while in Chitradurga district, 17 out of 33 samples, were positive (52\%) by PCR.

\subsection{Nucleotide sequence analysis}

A total of 70 samples were subjected for PCR by targeting B2L gene of ORFV, out of which, 42 samples were found positive for ORFV. The 16 samples, representing different regions of the Karnataka state were subjected for full length B2L gene nucleotide sequencing at $\mathrm{M} / \mathrm{s}$ Amnion Biotech Pvt Ltd. Bengaluru, Karnataka. However, out of 16 samples, full length sequencing of 1137bp nucleotides was achieved only in 6 samples and rest 10 samples yielded partial B2L gene sequences upon sequencing. Hence, during this study apart from full length phylogenetic analysis of 6 isolates, analysis of corresponding partial nucleotide sequences of all 16 isolates was carried out to understand the molecular epidemiology of ORFV isolates circulating in Karnataka. This consensus sequence was then further used for alignment with the published sequences of ORFV that are available in GenBank using NCBI Blast and CLUSTAL W (1.82) software and DNAstar software. The details of published sequences of ORFV used for phylogenetic analysis are listed in Table 1.

The nucleotide sequences obtained from the B2L gene PCR products of $1137 \mathrm{bp}$ (full length) and 250bp (partial length) of all 16 isolates of ORFV were initially aligned with the corresponding ORFV nucleotide sequences published. The full length nucleotide sequences of ORFV KVAFSU VMC-05, ORFV KVAFSU VMC19, ORFV KVAFSU VMC-20, ORFV KVAFSU VMC-36, ORFV KVAFSU VMC-42, and ORFV KVAFSU VMC-53, isolates shared 100 per cent homology with Assam/09 and Assam/10 isolates (GenBank accession number JN 8468834.1 and JQ 040300.1). The partial length nucleotide sequences of ORFV KVAFSU VMC-01, ORFV KVAFSU VMC-17, ORFV KVAFSU VMC-38, ORFV KVAFSU VMC-51, ORFV KVAFSU VMC-55, ORFV KVAFSU VMC-56, ORFV KVAFSU VMC-59, ORFV KVAFSU VMC-60, ORFV KVAFSU VMC-61 and ORFV KVAFSU VMC-63, isolates shared more than 99 per cent homology with Assam/10 isolates (GenBank accession number JQ 040300.1).
3.5 Phylogenetic analysis and sequence pair distance of Orf virus isolates

In the present study, all the 16 ORFV isolates were subjected for phylogenetic analysis along with published sequences of ORFV to understand the genetic similarities among the isolates. The nucleotide sequence pair distance of B2L gene for $16 \mathrm{ORFV}$ isolates with published ORFV sequences was determined. The sequence analysis was performed with the MegAlign program of Lasergene 6 package (DNAstar Inc. Madison, USA). Phylogenetic analyses of the $1137 \mathrm{bp}$ fragment of ORFV were conducted using MEGA version 6.06 using the maximum parsimony method with 1000 bootstrap replicates. The tree was constructed with the modules of the MEGA 6.06 Programme.

3.6 Phylogenetic analysis based on full length sequence of B2L gene of Orf virus isolates

The nucleotide sequence of full length gene isolates were compared with that of 36 sequences representing Indian and foreign isolates of PPVs including ORFV, Pseudo cow pox virus (PCPV) and Bovine papular stomatitis virus (BPSV), available in the database. The sequence analysis revealed that there was close relationship among the ORFV isolates worldwide. However, ORFVs obtained in this study were clustered separately from other members of the genus. The six isolates of both sheep and goats were grouped in a single cluster, indicating high level of genetic homology between these isolates. The isolates obtained in this study had highest sequence identity with Assam/09 and Assam/10 isolates (Figure 6)

Sequence analysis revealed high nucleotide (98.7-100\%) and amino acids (98.4-99.7\%) identity among Indian isolates maximum with Assam/09 \& Assam/10 isolates of ORFV (Figure 8). Sequence analysis with BPSV reference strain showed 84.3-84.5 per cent and 83.1-83.6 per cent sequence identity and with PCPV reference strain showed 94.4-94.7 per cent and 94.7-95.5 per cent sequence identity at the nucleotide and amino acid level respectively.

\subsection{Phylogenetic analysis based on partial length sequences (250 bp) of B2L gene of Orf virus isolates}

The partial sequences (250bp) of ORFV were compared with 16 sequences representing Indian and foreign isolates of PPVs including ORFV, PCPV and BPSV available in the database. The phylogenetic analysis revealed close relationship among the worldwide ORFV isolates. However, ORFVs obtained in this study were clustered separately from other members of the genus. Further, the analysis revealed that 16 isolates of both sheep and goats were grouped in a single cluster, indicating the high level of genetic relatedness between these isolates. The study isolates had highest genetic relationship with Assam/10 Muk/09 and caprine isolates (Figure 7). 
Table 1 Details of published sequences of B2L gene (full length) of parapoxviruses used in phylogenetic analysis.

\begin{tabular}{|c|c|c|c|c|c|}
\hline Sl. No. & Gene Origin & Collection year & Affected Species & Accession No. & Country of isolation \\
\hline 1. & ORFV-Assam/10 & 2010 & Goat & JN846834 & India \\
\hline 2. & ORFV-Assam/09 & 2009 & Goat & JQ040300 & India \\
\hline 3. & ORFV-Shanhjahnpur 82/04 & 2004 & Goat & DQ263303 & India \\
\hline 4. & ORFV-Mukteswar 59/05 & 2005 & Goat & DQ263304 & India \\
\hline 5. & ORFV-Muk/00 & 2000 & Goat & HM466933 & India \\
\hline 6. & ORFV-caprine & 2003 & Goat & AY278208 & USA \\
\hline 7. & ORFV-vaccine strain & 2003 & Goat & AY278209 & USA \\
\hline 8. & ORFV-SA00 & 2004 & Goat & AY386264 & USA \\
\hline 9. & ORFV-Nantou & 2007 & Goat & DQ904351 & Taiwan \\
\hline 10. & ORFV-Taiping & 2009 & Goat & EU327506 & Taiwan \\
\hline 11. & ORFV-Hoping & 2008 & Goat & EU935106 & Taiwan \\
\hline 12. & ORFV-A Brazil & 1980 & Goat & JN088053 & Brazil \\
\hline 13. & ORFV-NE2 & 1993 & Goat & JN088051 & Brazil \\
\hline 14. & ORFV-LiaoNing & 2010 & Goat & HQ694773 & China \\
\hline 15. & ORFV-Shanxi & 2009 & Goat & HQ202153 & China \\
\hline 16. & ORFV-HuB/2009 & 2009 & Goat & GU320351 & China \\
\hline 17. & ORFV-Korea/2009 & 2009 & Goat & GQ328006 & Korea \\
\hline 18. & ORFV-Mukteswar 67/04 & 2004 & Sheep & DQ263305 & India \\
\hline 19. & ORFV-Izatnagar 79/04 & 2004 & Sheep & DQ263306 & India \\
\hline 20. & ORFV-Muk/2009 & 2009 & Sheep & GU139356 & India \\
\hline 21. & ORFV-NZ2 & 1994 & Sheep & OVU06671 & New Zealand \\
\hline 22. & ORFV-ovine isolate & 2004 & Sheep & AY424970 & USA \\
\hline 23. & ORFV-IA82 & 1982 & Sheep & AY386263 & USA \\
\hline 24. & ORFV-D Brazil & 1992 & Sheep & JN088052 & Brazil \\
\hline 25. & ORFV-GanSu & 2009 & Sheep & HQ694772 & China \\
\hline 26. & ORFV-JS04 & 2006 & Sheep & GU903501 & China \\
\hline 27. & ORFV-Jilin & 2008 & Sheep & FJ808074 & China \\
\hline 28. & ORFV-Cam/09 & 2008 & Camel & GU460370 & India \\
\hline 29. & ORFV-Jodhpur & 2008 & Camel & GQ390365 & India \\
\hline 30. & ORFV-takin isolate & 2004 & Takin & AY424971 & USA \\
\hline 31. & ORFV-Musk ox & 2004 & Musk ox & AY424969 & USA \\
\hline 32. & PCPV-Tillquist & 2004 & Cow & AY424972 & Not available \\
\hline 33. & PCPV-VR634 & 2010 & Reindeer & GQ329670 & Not available \\
\hline 34. & ORFV-F92.849R & 2011 & Reindeer & JF773697 & Finland \\
\hline 35. & BPSV-Reference strain & 2004 & Calf & AY424973 & Not available \\
\hline 36. & BPSV-AR02 & 2004 & Calf & AY386265 & USA \\
\hline
\end{tabular}

Journal of Experimental Biology and Agriculture Science http://www.jebas.org 


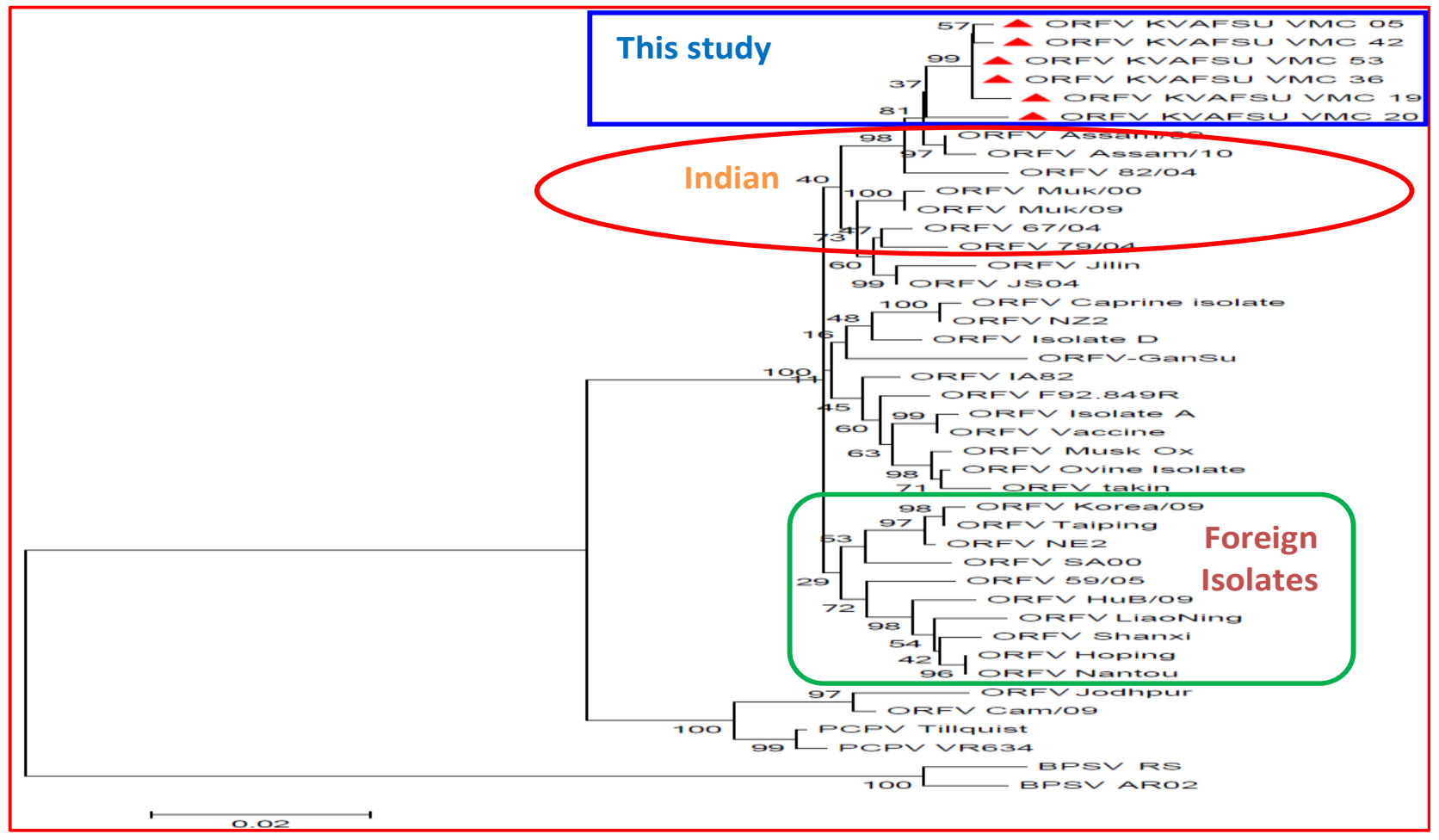

Figure 6 Phylogenetic analysis of different Orf viruses based on nucleotide sequences of full length B2L gene

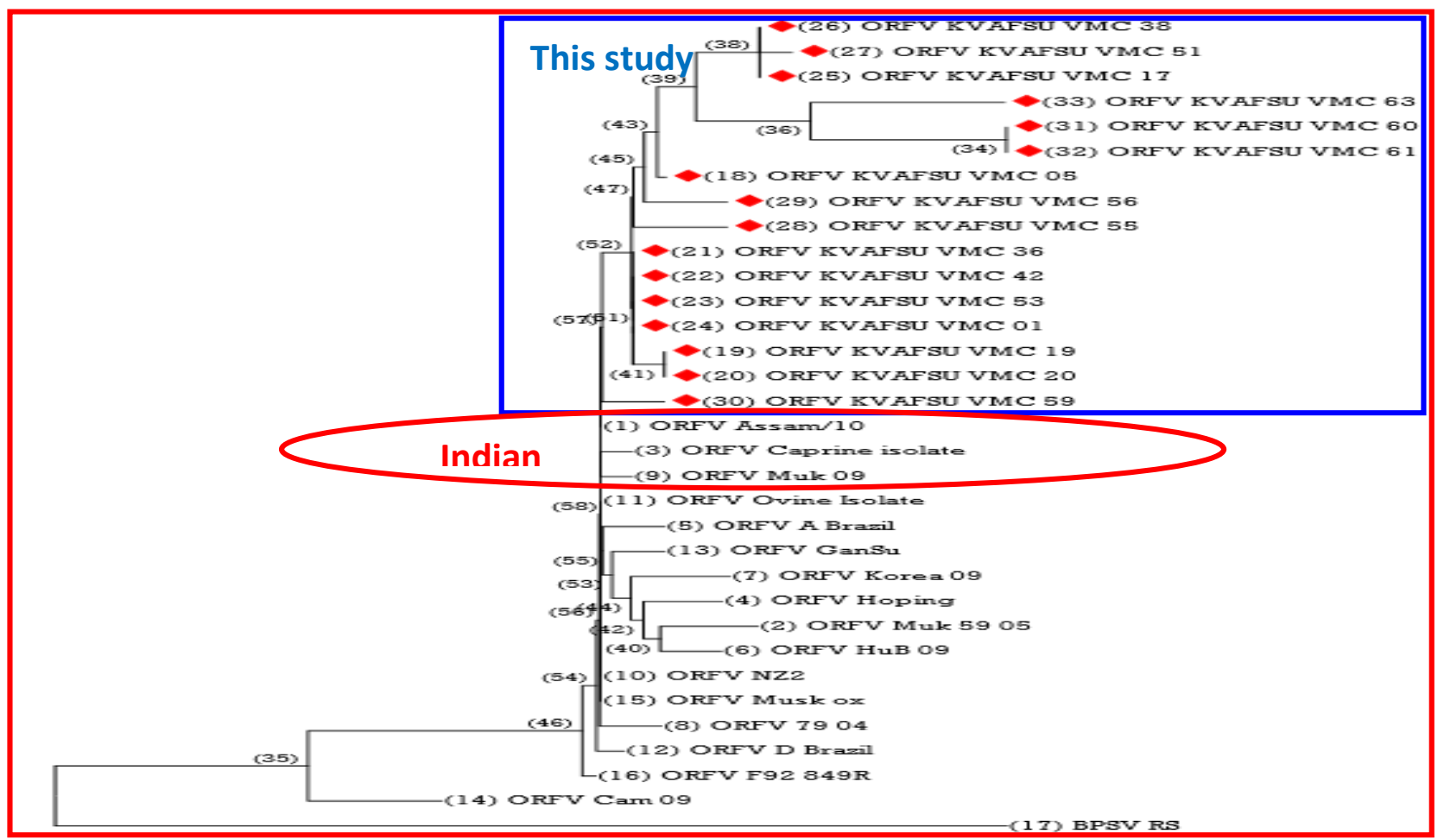

Figure 7 Phylogenetic analysis based on nucleotide sequence of partial B2L gene (MEGA version 5.04 and 1000 bootstrap replicates).

Journal of Experimental Biology and Agriculture Science http://www.jebas.org 


\begin{tabular}{|c|c|c|c|c|c|c|c|c|c|c|c|c|c|c|c|c|c|c|c|c|c|c|c|c|c|c|}
\hline & & & & & & & & & & & & Percent & identit & & & & & & & & & & & & & \\
\hline & 1 & 2 & 3 & 4 & 5 & 6 & 7 & 8 & 9 & 10 & 11 & 12 & 13 & 14 & 15 & 16 & 17 & 18 & 19 & 20 & 21 & 22 & 23 & 24 & & \\
\hline 1 & 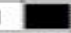 & 85.1 & 84.4 & 848 & 85.4 & 848 & 849 & 843 & 842 & 843 & 845 & 843 & 845 & 845 & 843 & 849 & 848 & 849 & 848 & 849 & 85.0 & 847 & 85.0 & 850 & 1 & BPSV_ARNO \\
\hline 2 & 166 & & 1977 & 98.2 & 98.5 & 982 & 979 & 97.8 & 97.6 & 978 & 98.0 & 978 & 98.0 & 98.0 & 97.7 & 979 & 980 & 99.1 & 975 & 989 & 99.1 & 982 & 97.9 & 963 & 2 & OREV -6704 \\
\hline 3 & 17.5 & 23 & & 98.7 & 978 & 97.5 & 97.1 & 98.4 & 982 & 98.1 & 98.6 & 96.4 & 98.6 & 98.4 & 966 & 972 & 97.4 & 97.4 & 959 & 97.9 & 98.1 & 969 & 97.1 & 945 & 3 & OREV 8204 \\
\hline 4 & 17.0 & 18 & 1.3 & & 98.4 & 97.7 & 97.5 & 99.2 & 990 & 990 & 99.4 & 992 & 99.4 & 99.7 & 97.4 & 97.6 & 978 & 98.0 & 975 & 983 & 98.5 & 977 & 97.7 & 960 & 4 & ORGV Assam 09 \\
\hline 5 & 163 & 15 & 22 & 16 & & 982 & 98.0 & 980 & 978 & 978 & 982 & 980 & 98.2 & 982 & 97.5 & 982 & 98.4 & 980 & 982 & 98.5 & 98.7 & 983 & 99.0 & 960 & 5 & OREV-1AB2 \\
\hline 6 & 17.0 & 19 & 25 & 23 & 1.8 & & 980 & 973 & 97.1 & 97.3 & 97.4 & 973 & 97.4 & 97.4 & 969 & 982 & 962 & 97.9 & 978 & 962 & 983 & 97.4 & 97.6 & 946 & 6 & ORFV- Buk 5905 \\
\hline 7 & 169 & 21 & 3.0 & 25 & 21 & 21 & & 97.1 & 969 & 969 & 973 & 97.1 & 97.3 & 973 & 96.7 & 980 & 98.5 & 97.7 & 98.4 & 97.9 & 98.1 & 97.9 & 98.1 & 952 & 7 & ORFV-SWOO \\
\hline 8 & 17.6 & 22 & 1.5 & 0.8 & 21 & 28 & 30 & & 99.5 & 967 & 99.8 & 99.6 & 99.8 & 989 & 968 & 97.4 & 97.5 & 97.5 & 973 & 97.9 & 98.1 & 97.3 & 973 & 945 & 8 & OREV_KVAFSU_MUC_06 \\
\hline 9 & 17.8 & 24 & 18 & 10 & 22 & 30 & 3.1 & 0.5 & & 96.5 & 99.5 & 995 & 99.6 & 98.8 & 967 & 972 & 97.4 & 97.4 & 97.1 & 97.7 & 979 & 97.1 & 97.1 & 94.4 & 9 & ORPV_KVAFSU_MIC_19 \\
\hline 10 & 17.6 & 22 & 20 & 10 & 22 & 28 & 3.1 & 1.3 & 15 & & 989 & 98.7 & 989 & 98.8 & 968 & 972 & 973 & 975 & 970 & 97.7 & 97.9 & 97.1 & 97.1 & 94.4 & 10 & OREV_KVAFSU_MIC_2O \\
\hline 11 & 1773 & 21 & 1.4 & 0.6 & 19 & 26 & 28 & 02 & 0.4 & 12 & & 998 & 1000 & 99.1 & 970 & 975 & 977 & 97.7 & 97.4 & 981 & 982 & 97.4 & 97.4 & 947 & 11 & OREV_KVAFSU_WC__36 \\
\hline 12 & $2 \quad 17.6$ & 22 & 1.5 & 0.8 & 21 & 28 & 30 & 0.4 & 0.5 & 1.3 & 02 & & 99.8 & 98.9 & 968 & 97.4 & 97.5 & 97.5 & 973 & 97.9 & 96.1 & 97.3 & 97.3 & 94.5 & 12 & OREV_KVAFSU_MAC_42 \\
\hline 13 & 173 & 21 & 1.4 & 0.6 & 1.9 & 26 & 28 & 02 & 0.4 & 12 & 0.0 & 02 & & 99.1 & 970 & 97.5 & 977 & 97.7 & 97.4 & 98.1 & 982 & 97.4 & 97.4 & 947 & 13 & OREV_KVAFSU_WMC_53 \\
\hline 14 & 17.3 & 21 & 1.5 & 03 & 19 & 26 & 28 & 1.1 & 12 & 12 & 09 & 1.1 & 0.9 & & 972 & 97.4 & 975 & 97.7 & 973 & 98.1 & 982 & 97.4 & 97.4 & 947 & 14 & OREV-issam 10 \\
\hline 15 & 17.6 & 23 & 3.5 & 26 & 25 & 32 & 33 & 32 & 33 & 32 & 3.1 & 32 & 3.1 & 29 & & 97.4 & 970 & 97.4 & 95.7 & 97.4 & 97.6 & 97.6 & 97.1 & 94.1 & 15 & OREVGanSu \\
\hline 16 & 169 & 21 & 29 & 24 & 19 & 19 & 21 & 27 & 29 & 29 & 25 & 27 & 25 & 27 & 25 & & 98.1 & 97.4 & 97.6 & 97.9 & 98.1 & 973 & 97.4 & 94.4 & 16 & ORFVHoping \\
\hline 17 & 170 & 21 & 27 & 22 & 1.6 & 19 & 15 & 25 & 27 & 28 & 23 & 25 & 23 & 25 & 3.1 & 20 & & 97.8 & 99.6 & 980 & 982 & 978 & 980 & 960 & 17 & OREVisolate nE2 Brasil 1993 \\
\hline 18 & 169 & 09 & 2.5 & 21 & 21 & 21 & 23 & 25 & 27 & 25 & 23 & 25 & 23 & 23 & 27 & 26 & 22 & & 97.4 & 98.8 & 989 & 97.8 & 975 & 96.1 & 18 & OREV-Lathagar 7904 \\
\hline 19 & 17.0 & 25 & 32 & 25 & 19 & 22 & 16 & 28 & 30 & 3.1 & 26 & 28 & 26 & 28 & 3.3 & 24 & 0.4 & 27 & & 975 & 97.7 & 97.9 & 98.1 & 949 & 19 & ORFV-Worea 2009 \\
\hline 20 & 16.9 & 1.1 & 21 & 17 & 1.5 & 19 & 21 & 21 & 23 & 23 & 20 & 21 & 20 & 20 & 26 & 21 & 21 & 12 & 25 & & | 99.8 & 98.1 & 97.9 & 962 & 20 & OPEVYHUK 2000 \\
\hline 21 & 167 & 0.9 & 20 & 15 & 13 & 1.7 & 20 & 20 & 21 & 21 & 18 & 20 & 1.8 & 1.8 & 24 & 20 & 19 & 1.1 & 23 & 0.2 & & 981 & 98.1 & 963 & 21 & OFFVAluk 2009 \\
\hline 22 & 17.1 & 19 & 32 & 23 & 1.7 & 26 & 21 & 28 & 30 & 30 & 26 & 28 & 26 & 26 & 24 & 28 & 22 & 22 & 21 & 20 & 20 & & 1988 & 963 & 22 & OREVACZ \\
\hline 23 & 168 & 21 & 30 & 23 & 1.0 & 24 & 20 & 28 & 30 & 30 & 26 & 28 & 26 & 26 & 3.0 & 26 & 21 & 25 & 20 & 21 & 20 & 12 & & 1963 & 23 & ORFV-Vacoine strain \\
\hline 24 & 155 & 48 & 58 & 52 & 52 & 5.6 & 50 & 5.7 & 59 & 59 & 5.5 & 5.7 & 55 & 5.5 & 6.1 & 59 & 52 & 5.1 & 53 & 5.0 & 48 & 49 & 49 & & 24 & PCPV-Tillquist \\
\hline & 1 & 2 & 3 & 4 & 5 & 6 & 7 & 8 & 9 & 10 & 11 & 12 & 13 & 14 & 15 & 16 & 17 & 18 & 19 & 20 & 21 & 22 & 23 & 24 & & \\
\hline
\end{tabular}

Figure 8: Nucleotide sequence identity of different parapox viruses based on full length B2L gene (Determined by Clustal W method in the MegAlign program of Lasergene (DNASTAR), Upper right triangle is the nucleotide, sequence identities, Lower left triangle is the divergence identities)

The sequence analysis revealed high nucleotide (98.7-100\%) and amino acids (83.3-98.8\%) identity among Indian isolates maximum with Assam/10 and Muk /09 isolates of ORFV. The sequence analysis with BPSV reference strain showed 84.2-84.5 per cent and 77.4-85.7 per cent sequence identity at the nucleotide and amino acid levels respectively (Figure 9).

Multiple alignment of deduced amino acid sequences was carried out and compared with that of 36 sequences representing ORFV, PCPV and BPSV available in the NCBI database. It showed many substitutions dispersed all along the length of the protein and the major amino acid substitutions were noticed at three unique positions of A41T, A126T and D294N in coding region of B2L protein of these isolates (Figure10). The isolate ORFV KVAFSU VMC-05 showed five deduced amino acid sequences substitutions at E22A, A41T, A127T, D295N, and I377E in coding region of B2L protein of this isolate. The ORFV KVAFSU VMC-19 had the four substitutions at A41T, A127T, D217E, and D295N in coding region of $\mathrm{B} 2 \mathrm{~L}$ protein of this isolate.The isolate $\mathrm{ORFV}$ KVAFSU VMC-20 showed seven substitutions at E22D, A24E, A41T, A53T, A127T, K231Q, and D295N in coding region of $\mathrm{B} 2 \mathrm{~L}$ protein of this isolate. The ORFV KVAFSU VMC-36 showed three substitutions at A41T, A127T, and D295N in coding region of B2L protein of this isolate. The ORFV KVAFSU VMC42 the full length sequences showed four substitutions at I7F,
A41T, A127T, and D295N in coding region of B2L protein of this isolate. The ORFV KVAFSU VMC-53 showed three substitutions at A41T, A127T, and D295N in coding region of B2L protein of this isolate (Figure 10).

\section{Discussions}

Orf also known as contagious ecthyma is an acute, debilitating and economically important zoonotic viral skin disease that affects sheep, goat and other domesticated and wild ruminants. The disease also has a significant economic impact on rural communities that are predominantly relying on livestock farming for their livelihood. Although the disease is mild and self limiting, it can be fatal and persistent in lambs and kids. Severe facial and oral lesions in lambs may interfere with suckling, lesions on the udder may result in the abandonment of offspring and foot lesions can cause transient lameness. Infected animals fail to thrive and more prone for secondary bacterial and fungal infections. The disease is endemic in India and outbreaks are reported in sheep and goats in different agro-climatic regions (Bora et al., 2012).

The ability of ORFV to cross infect other species of animals including camels, Japanese serows, musk ox and reindeer have also been reported. Higher mortality due to Orf infections has been noticed in some of these animals whilst the severity of the lesion may also result in slaughter of animals on the grounds of 


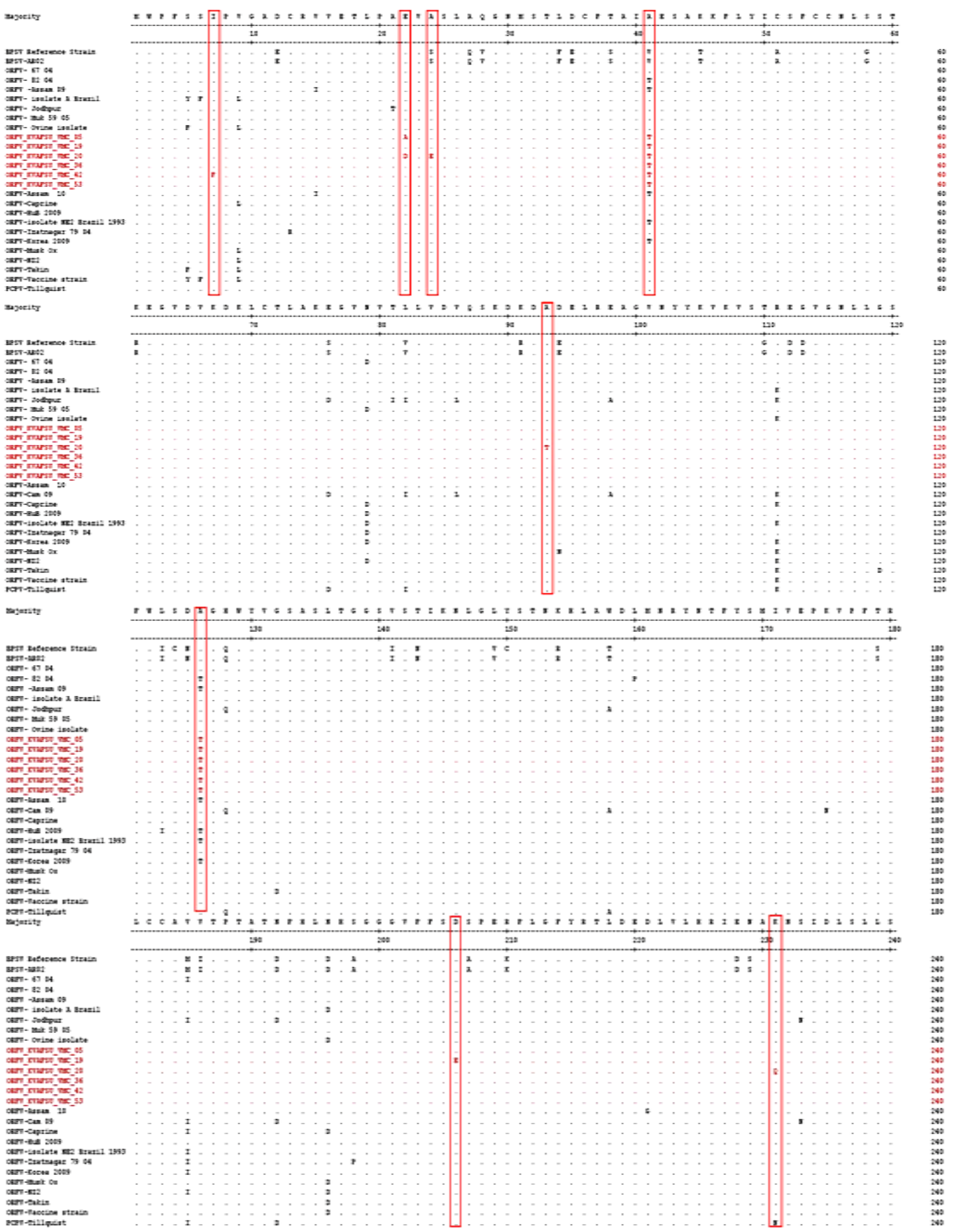

Journal of Experimental Biology and Agriculture Science http://www.jebas.org 

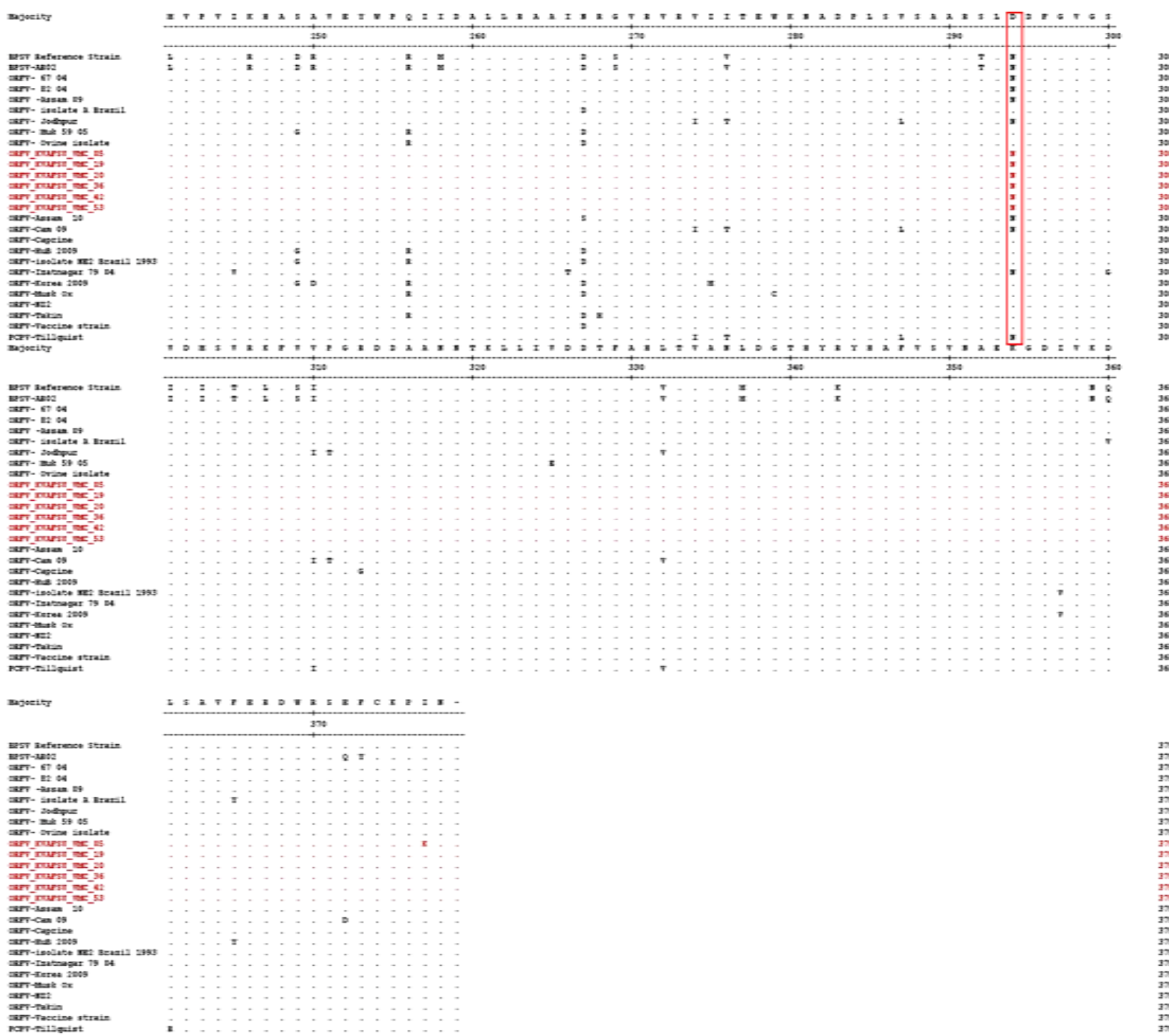

Figure 10 Multiple alignment of deduced amino acids sequences of different PPVs of full length B2L gene

welfare (Inoshima et al., 2002; Howsawi et al., 2004). The ORFV has its own immune evasion mechanisms which helps to adapt and replicate in the presence of an active immune response (Azwai et al.,1995; Hosamani et al., 2009).

In the present study, samples were collected from high density sheep and goat populated districts with emphasis on severe outbreaks with repeated reoccurrence of the disease in the same herd and flocks. The disease was severe in lambs/kids and deaths were seen in some flocks, where the pain in the oral cavity, making them unable to take milk or feed, leading to starvation and deaths. These results suggested significant economic impact of ORFV on poor farmers due to reduction in production. Similar disease pattern in the sheep and goats has been reported from other Indian states such as Andhra Pradesh, Assam, Bihar, Gujarat, Haryana, Himachal Pradesh, Jammu and Kashmir, Karnataka, Madhya Pradesh, Maharashtra, Meghalaya, Orissa, Punjab, Rajasthan, Tamil Nadu, Uttarakhand, Uttar Pradesh, and West Bengal (Chandranaik et al., 2005; Hosamani et al., 2009).

Clinically proliferative lesions were noticed on the skin of lips, oral mucosa, muzzle, lower jaw, eye, ear and around the nostrils. Initially, lesions appeared as erythematous spots or swelling, which was followed by formation of papules and pustules with a yellowish creamy appearance. These papules progressed to develop into scabs. These findings were in agreement with that of Chandranaik et al., (2005) and Bora et al., (2012). 
Upon inoculation of samples in ECE by CAM route yielded the characteristic white, firm and necrotic pock lesions. This proves that virus could be easily grown in chick embryos. Present study finds that chicken embryos are better indicator system compared to cell cultures for adoption of ORFV. Further ECE are easily available and inoculation is rapid and simple when compared to skills and equipment required for the isolation of ORFV in cell cutlers. More over the results could be obtained within five days compared to cell cultures which need 14 days to develop CPE as opined by Mohamed et al. (2010).

In the present study, standardization of PCR was carried out for highly conserved region of B2L gene of ORFV. The B2L gene has been routinely used as the detection target of ORFV in many diagnostic laboratories worldwide (Hosamani et al., 2006). The B2L region is chosen in order to ensure adequate fidelity of the assay and reduce interference due to genetic mutation. Sullivan et al. (1994) have also described that B2L gene is valuable target for the detection, especially when ORFV particles are very less in clinical samples. Kottaridi et al. (2006) and Hosamani et al. (2006) have also found that B2L gene is highly specific and the most important gene for the ORFV detection by PCR.

The B2L gene sequences of ORFV, a major envelope protein antigen has been widely used by many researchers as a target for the development of nucleic acid based diagnostics and molecular epidemiology of Orf virus infections. It provides epidemiologically important information and large number of sequence data is available in the GenBank that can be used for epidemiological and phylogenetic analyses of many parapoxviruses (Bora et al., 2012). Phylogenetic analysis of the sequences of 1137 bp full length and 250 bp fragment of B2L gene were conducted. The full length sequence data of B2L gene of the six isolates clearly indicated that they are indeed orf viruses. Comparison of isolates obtained in this study with Indian and foreign isolates, revealed close relationship among the worldwide ORFV isolates. However, current ORFVs clustered separately from other members of the genus and these isolates were genetically most closely related to Assam/09 and Assam/10 ORFV isolates. Six ORFV isolates of both sheep and goats were grouped in a single cluster indicating high level of genetic homology between these isolates when compared with other PPVs including ORFV, PCPV and BPSV available in the database. This is consistent with the fact that central region of parapoxviruses where B2L gene is located and targeted in this study is generally well conserved in comparison to the terminal regions as described by Hosamani et al. (2006).

The partial sequences of B2L gene of sixteen isolates were compared with thirteen other Indian and foreign isolates and revealed that there was close relationship among the worldwide
ORFV isolates. However, ORFVs were clustered separately from other members of the genus as reported elsewhere. Isolates obtained in this study were closely related with the Assam/10 and Muk/09 isolates. Interestingly, all the 16 isolates from the study were grouped together, indicating very high level of genetic homology in contrast with Assam and Muktheswar isolates which were intermixed with the foreign isolates.

Phylogeneticlaly ORFV isolates were clustered with north Indian virus isolates. Although the phylogenetic analysis indicates the hypothetical origin of the virus isolates, it is difficult to determine the exact origin of the viruses that were introduced into the current outbreaks. However these data suggests that the circulation of ORFV might be due to the migration of sheep and goat flocks from neighboring states in search of fodder and animal fairs, which are frequently held in various states of country.

The detailed molecular analysis of the ORFV sequences is the need of the hour to clarify epidemiology and transmission of disease in these outbreaks that has prompted to seek more information about the mutations between the ORFV isolates under this study. For this multiple sequence alignment was carried out that showed three unique amino acid substitutions (A41T, A126T and $\mathrm{D} 294 \mathrm{~N}$ ) in the coding region of B2L protein of these six isolates compared to other PPVs. It was also demonstrated that many substitution of amino acids including unique amino acids specific for Orf viruses are being dispersed randomly along the B2L protein of different ORFV isolates (Hosamani et al., 2006). Similar observations have also been made by Bora et al., (2012). However, no other unique amino acid substitutions were observed in the protein sequence that would probably reflect the host specificity of an individual ORFV. In broader sense, it implies that PPVs are antigenically and genetically closely related (Fleming et al., 1993).

\section{Conclusion}

The Orf disease is endemic in Karnataka. The restriction of movement of animals especially in migratory flocks during the phase of disease is necessary to prevent the spread of the disease. The PCR assay can be used to detect and differentiate parapoxvirus infections in small ruminants. For further confirmation the purified PCR products can be sequenced and analysed comparing with the published sequences.

\section{Acknowledgement}

The authors are highly thankful for the Karnataka Veterinary, Animal and Fisheries Sciences University for providing facilities to carry out the research work at Veterinary College, Hebbal, Bengaluru. 


\section{Conflict of Interest}

The authors declare that there is no conflict of interest regarding the publication of this research paper.

\section{References}

Azwai SM, Carter SD, Woldehiwet Z (1995) Immune responses of the camel (Camelus dromedarius) to contagious ecthyma (Orf) virus infection.Veterinary Microbiology 47: 119-131

Bora DP, Venkatesan G, Bhanuprakash V, Balamurugan V, Prabhu M, Siva sankar MS, Yogisharadhya R (2011) Taq Man real-time PCR assay based on DNA polymerase gene for rapid detection of orf infection. Journal of Virological Methods 78 : 249-252.

Bora DP, Barman NN, Das SK, Bhanuprakash V, Yogisharadhya R, Venkatesan G Kumar A, Rajbongshi G, Khatoon E, Chakraborty A, Bujarbaruah KM (2012) Identification and phylogenetic analysis of Orf viruses isolated from outbreaks in goats of Assam, a north - eastern state of India. Virus Genes 45: 98-104.

Caravaglio JV , Khachemoune A (2017) Orf Virus Infection in Humans: A Review with a focus on advances in diagnosis and treatment. Journal of Drugs in Dermatology 16: 684-689.

Chandranaik BM, Harish BR, Mallikarjun patil, Byregowda SM, Mamtha GS, Jayakumar SR, Krishnappa G (2005) Outbreak of Orf in sheep: an investigation. Intas Polivet : 66-67.

Delhon G, Tulman ER, Afonso CL (2004) Genomes of the Parapoxviruses: Orf virus and bovine papular stomatitis virus. Journal of Virology 78: 168-177

Fleming SB, Block J, Fraser KM, Mercer AA, Robinson AJ (1993) Conservation of gene structure and arrangement between vaccinia virus and Orf virus. Virology 195: 175-184.

Gelaye E, Achenbach J E, Jenberie S, Gelagay A, Belay A, Loitsch MY, Reingard G, Adama D, Charles EL (2016) Molecular charcetrization of orf virus from sheep and goats in Ethiopia,2008-2013. Virology Journal 13:34.

Hosamani M, Bhanuprakash V, Scagliarini A, Singh RK (2006) Comparative sequence analysis of major envelope protein gene (B2L) of Indian Orf viruses isolated from sheep and goats. Veterinary Microbiology 116: 317-324.

Hosamani M, Yadav S, Kallesh DJ, Mondal B, Bhanuprakash V, Singh RK, (2007) Isolation and characterization of an Indian Orf virus from goats. Zoonoses and Public Health 54: 204-208.

Hosamani M, Scagliarini A., Bhanuprakash V, Mcinnes CJ, Singh RK (2009) Orf: an update on current research and future perspectives. Expert Review of Anti- infective Therapy 7: 879893.

Housawi FMT, Abu-Elzein E, Gameel A, Mustafa M, Nettleton P (2004) Severe Auzsyk infection in one-month-old camel calves (Camelus dromedarius). Veterinary Archives 74: 467-474

Inoshima Y, Morooka A, Sentsui H (2000) Detection and diagnosis of Parapoxvirus by the PCR. Journal of Virological Methods 84: 201-208.

Inoshima Y, Murakami K, Wu D, Sentsui H (2002) Characterization of parapoxviruses circulating among wild Japanese serows (Capricornis crispus). Microbiology and Immunology 46: 583-587

Kottaridi C, Nomikou K, Teodori 1, Savini G, Lelli R, Markoulatos P, Mangana O (2006) Phylogenetic correlation of Greek and Italian Orf virus isolates based on VIR gene. Veterinary Microbiology 16: 310-316.

Kumar AA, Shivachandra SB, Biswas A, Singh VP, Singh VP, Srivastava SK (2004) Prevalent serotypes of Pasteurella multocida isolated from different animal and avian species in India. Veterinary Research Communication 28 : 657-667.

Mckeever DJ, Reid HW, Inglis NF, Herring AJ (1987) A qualitative and quantitative assessment of the humoral antibody response of the sheep to Orf virus infection. Veterinary Microbiology 15: 229-241.

Mohamed, Mahmoud A, Khaled Abdelrahman A, Hatem Soliman (2010) Molecular and virological studies on contagious pustular dermatitis isolates from Egyptian sheep and goats. Research in Veterinary Science 89: 290-294.

OIE (2007) Manual of diagnostic tests and vaccines for terrestrial animals (mammals, birds and bees). Office International des Epizooties, Paris, France. Pp739-750.

Saitou N, Nei M (1987) The neighbor joining method: a new method for reconstructing phylogenetic trees. Molecular Biology and Evolution 4: 406-425.

Spyrou V, Valiakos G (2015) Orf virus infection in sheep and goats. Veterinary Microbiology 181: 178-182.

Sullivan JT, Mercer AA, Fleming SB, Robinson AJ (1994) Identification and characterization of an Orf virus homologue of the vaccinia virus gene encoding the major envelope antigen p37K.Virology 202: 968-973

Thompson JD, Higgins DG, Gibson TJ, Clustal W (1994) Improving the sensitivity of progressive multiple sequence alignment through sequence weighting, position-specific gap penalties and weight matrix choice. Nucleic Acids Research 22:4673-4680. 